\title{
NeuroRegulation
}

\section{Proceedings of the 2020 ISNR Annual Conference (Virtual): Poster Presentations}

\author{
Selected Abstracts of Conference Poster Presentations at the 2020 International Society for \\ Neurofeedback and Research (ISNR) 28th Annual Conference, Miami, Florida, USA
}

Citation: International Society for Neurofeedback and Research. (2020). Proceedings of the 2020 ISNR Annual Conference (Virtual): Poster Presentations. NeuroRegulation, 7(4), 173-183. https://doi.org/10.15540/nr.7.4.173

Copyright: $\odot$ 2020. ISNR. This is an Open Access article distributed under the terms of the Creative Commons Attribution License (CC-BY).

\section{A Way of Treating Very Severe Psychiatric Clients Who Showed Extremely High Amplitude of Theta Waves \\ Miyako Tazaki \\ Faculty of Medicine, Toho University, Tokyo, Japan}

Some of the clients who had a long history of psychiatric disorders showed extremely high amplitude of theta waves, such as more than 60 to $200 \mu \mathrm{V}$, so we have developed an effective procedure to treat them with neurofeedback. These clients usually had a long history of shopping doctors for more than five years for not having improved with only psychiatric drugs, and they had usually been diagnosed with various psychiatric disorders, anxiety, developmental disorders, and schizophrenia. With my clinical experience, a high amplitude of theta waves may be caused by the inflammation of brain cells induced by a leaky brain induced by leaky gut syndrome caused by gluten allergies, ticks, infection diseases, electromagnetic wave, and PTSD with dissociation disorders. Among 658 clients, $75 \%$ of them had been diagnosed as to have the leaky gut syndrome. Therefore, in our practice, we at first evaluate brain waves, secondly conduct counselling on their personal and family histories, and thirdly ask about their lifestyle, food intake, exercise habits, and personal relationships, then educate on nutrition and exercises, as well as conduct cognitive behavior therapy. Then, we usually refer them first to an internal medicine doctor who could diagnose and treat leaky gut syndrome, prescribe medication as well as supplement to detoxify and nourish the brain, and, if necessary, refer to a clinician of Self-Ego Unification Therapy invented in Japan to treat dissociation disorders. For clients who had recovered from their leaky gut syndrome, their amplitude of theta waves decreased to less than 20 $\mu \mathrm{V}$, and within less than five sessions they lost their abnormal symptoms. In our experience, three clients who diagnosed as schizophrenia got jobs.
Our recent procedure is first to eliminate factors to decrease theta waves, and then to provide NFT being backed up by two theories depending on their emerging area of psychiatry; one is the neuroinflammation theory that psychiatric disorders are caused by inflammation of brain cells, and the other is the connectome disorder theory that psychiatric disorders are caused by disorders of brain connectomes.

\section{References}

Marques, T. R., Ashok, A. H., Pillinger, T., Veronese, M., Turkheimer, F. E., Dazzan, P., ... Howes, O. D. (2019). Neuroinflammation in schizophrenia: Meta-analysis of in vivo microglial imaging studies. Psychological Medicine, 49(13), 2186-2196. https://doi.org/10.1017/s0033291718003057

Narr, K. L., \& Leaver, A. M. (2015). Connectome and schizophrenia. Current Opinion in Psychiatry, 28(3), 229-235. https://doi.org/10.1097/yco.0000000000000157

\section{Affective Virtual Reality Game for Depression Symptoms Detection Using \\ Psychophysiological and Behavioral Measures Christopher Baxter ${ }^{1}$, Estate Sokhadze ${ }^{2}$, and Yi (Joy) $\mathrm{Li}^{1}$ ${ }^{1}$ Kennesaw State University, Kennesaw, Georgia, USA \\ ${ }^{2}$ University of South Carolina School of Medicine in Greenville, Greenville, South Carolina, USA}

Since its inception, virtual reality (VR) technology has become not only an important medium of entertainment but also a useful tool in therapeutic intervention research. VR provides a fully immersive experience with the possibility of triggering strong and more specific emotional reactions, all within a safe environment. While this has led to interests in applications of VR as a therapeutic intervention (Freeman et al., 2018; Grochowska, Jarema, \& Wichniak, 2019), the application of VR as a diagnosing tool of mental disorders has not been thoroughly investigated yet. In our current research study, we have developed an affective VR system that aims to induce different types of emotional 
reactions in users. By instilling a sense of presence in the users through immersion and interaction (Baños et al., 2005; Sugiura, 2013), the system is able to impact on current user emotions. Three emotional-laden VR game scenes were carefully designed and developed with controlled variations in order to elicit targeted emotions. Physiological signals collected from an E4 wearable device (Empatica, Boston, MA) were used to monitor and analyze the dynamic emotional status. Based on our previous research (Li, Elmaghraby, El-Baz, \& Sokhadze, 2015; Sokhadze et al., 2017), we assume these emotional states will be different between the emotionally impaired groups and the control group. A mini psychomotor VR game followed immediately after each scene and was designed to test users' motor reaction time under the emotional impact. A pilot study has been conducted where participants divided into two categories: those with self-reported depression symptoms and those without any known mental illness or any depression symptoms. Results from collected postexperiment surveys show that the three affective game scenes are able to trigger targeted emotional reactions in all participants. Recorded physiological data shows that participants with self-reported depression symptoms exhibited higher skin conductance levels (SCL) and higher heart rates than individuals with no known mental illnesses symptoms. Recorded VR game exposure times show that participants with self-reported depression took longer to complete each scene than individuals with no known mental illnesses. The times necessary to complete the VR game were similar across both categories.

\section{References}

Baños, R. M., Botella, C., Alcañiz, M., Liaño, V., Guerrero, B., \& Rey, B. (2005). Immersion and emotion: Their impact on the sense of presence. CyberPsychology \& Behavior, 7(6), 734741. https://doi.org/10.1089/cpb.2004.7.734

E4 wristband [EDA/GSR sensor, real-time physiological signals]. (n.d.). Boston, MA: Empatica. Retrieved February 10, 2020, from https://www.empatica.com/research/e4

Freeman, D., Haselton, P., Freeman, J., Spanlang, B., Kishore, S., Albery, E., ... Nickless, A. (2018). Automated psychological therapy using immersive virtual reality for treatment of fear of heights: A single-blind, parallel-group, randomised controlled trial. The Lancet Psychiatry, 5(8), 625632. https://doi.org/10.1016/s2215-0366(18)30226-8

Grochowska, A., Jarema, M., \& Wichniak, A. (2019). Virtual reality-A valuable tool to advance treatment of mental disorders. Archives of Psychiatry and Psychotherapy, 21(1), 65-73. https://doi.org/10.12740/app/101654

Li, Y., Elmaghraby, A. S., El-Baz, A., \& Sokhadze, E. M. (2015, December). Using physiological signal analysis to design affective VR games. 2015 IEEE International Symposium on Signal Processing and Information Technology (ISSPIT), Abu Dhabi, United Arab Emirates. https://doi.org/10.1109 /isspit.2015.7394401
Sokhadze, E. M., Casanova, M. F., Kelly, D. L., Sokhadze, G. E., Li, Y., Elmaghraby, A. S., \& El-Baz, A. S. (2017). Virtual reality with psychophysiological monitoring as an approach to evaluate emotional reactivity, social skills, and join attention in autism spectrum disorder. In M. F. Casanova, A. El-Baz, \& J. S. Suri (Eds.), Autism Imaging and Devices (1st ed., Chapter 18). Boca Raton, FL: CRC Press. https://doi.org/10.1201 19781315371375-24

Sugiura, M. (2013). A cognitive neuroscience approach to self and mental health. In J. Wu (Ed.), Biomedical Engineering and Cognitive Neuroscience for Healthcare: Interdisciplinary Applications (pp. 1-10). Hershey, PA: IGI Global. https://doi.org/10.4018 /978-1-4666-2113-8.ch001

\section{Effect of Audio-Visual Brain Entrainment on Anxiety, General Health, Stress, Quality of Sleep and Work Productivity and Activity Impairment: A Pilot Study with Telemarketers Rodolfo Borges Parreira ${ }^{1}$, Noemy Ferreira de Castro', Maria Luisa Ramos Mendes ${ }^{1}$, Afonso Inoue Shiguemi Salgado ${ }^{2}$, Patrick Porter ${ }^{2}$, and Francisco Cidral-Filho ${ }^{2}$ ${ }^{1}$ Residence Program in Manipulative, Complementary and Integrative Physical Therapy, Salgado Institute of integral Health/Philadelphia University Center, Londrina, Brazil \\ ${ }^{2}$ Integrative Health School, USA}

The objective of this study was to investigate the effect of audio-visual brain entrainment on anxiety, general health, stress, quality of sleep, and work productivity and activity impairment of telemarketers. The study was conducted at the Salgado Institute of Integrative Health, Londrina, Paraná, Brazil, and the protocol was approved by the Institutions Ethics Committee. Sample size consisted of 13 telemarketers ( 3 males and 10 females). Audiovisual brain entrainment was delivered with a BrainTap headset (BrainTap, New Bern, NC) in 20min sessions, three times a week for 6 weeks. The sessions consisted of binaural beats at 18 to $0.5 \mathrm{~Hz}$, isochronic tones at 18 to $0.5 \mathrm{~Hz}$, and visual entrainment through light-emitting diodes at the wavelength of $470 \mathrm{~nm}$ that were placed on a visor flickering at 18 to $0.5 \mathrm{~Hz}$ over the eyes of the participants. The following questionnaires were applied at baseline and after 6 weeks: The Hamilton Anxiety Rating Scale (HAM-A) that measures the severity of anxiety symptoms; the General Health Questionnaire (GHQ-12), a screening device for identifying minor psychiatric disorders; the Perceived Stress Scale (PSS-10), the most widely used psychological instrument for measuring the perception of stress; the Pittsburgh Quality of Sleep Index (PQSI), that scores sleep quality; and the Work Productivity and Activity Impairment Questionnaire (WPAl), that measures impairments in work and activities. Audio-visual brain entrainment positively affected all scores: HAM-A (22.95\%); GHQ-12 (10.93\%); PSS-10 (16.86\%); PQSI 
(14.51\%); as well as WPAI (absenteeism, 41.66\%; presenteeism, $56.25 \%$; work productivity, $56.22 \%$; and activity Impairment due to health, $76 \%$ ).

Conclusion. Although results did not achieve statistical significance when compared to baseline, audio-visual brain entrainment positively affected scores related to anxiety, general health, stress, quality of sleep, as well as work productivity and activity impairment of telemarketers. A larger sample size study is necessary to statistically confirm the effects of audio-visual brain entrainment.

\section{References}

Chaieb, L., Wilpert, E. C., Reber, T. P., \& Fell, J. (2015). Auditory beat stimulation and its effects on cognition and mood states. Frontiers in Psychiatry, 6, $70 . \quad \mathrm{https} / / /$ doi.org /10.3389/fpsyt.2015.00070

Derner, M., Chaieb, L., Surges, R., Staresina, B. P., \& Fell, J. (2018). Modulation of item and source memory by auditory beat stimulation: A pilot study with intracranial EEG. Frontiers in Human Neuroscience, 12, 500. https://doi.org /10.3389/fnhum.2018.00500

Gao, X., Cao, H., Ming, D., Qi, H., Wang, X., Wang, X., ... Zhou, P. (2014). Analysis of EEG activity in response to binaural beats with different frequencies. International Journal of Psychophysiology., 94(3), 399-406. https://doi.org /10.1016/j.ijpsycho.2014.10.010

Gao, J., Fan, J., Wu, B. W., Zhang, Z., Chang, C., Hung, Y.-S., ... Sik, H. H. (2016). Entrainment of chaotic activities in brain and heart during MBSR mindfulness training. Neuroscience Letters, 616, 218-223. https://doi.org/10.1016 /j.neulet.2016.01.001

\section{Effects of Neurofeedback on \\ Electroencephalographic Functional \\ Connectivity in Children with Specific Learning Disorder with Impairment in Reading Lucero Albarran \\ Universidad Nacional Autónoma de México, Mexico City, Mexico}

Specific learning disorder with impairment in reading (RD) is a neurodevelopmental disorder characterized by alterations of the normal pattern to acquire reading academic skills, including the ability to read words accurately and fluently with reading comprehension. Several EEG studies have reported abnormalities in children with RD. The most frequently reported is a higher theta absolute power and lower alpha activity compared to children with typical development of the same age. Regarding the interhemispheric coherence measure, children with $\mathrm{RD}$ show higher values in delta $(1.5-3.5 \mathrm{~Hz})$, theta $(4.0-7.5 \mathrm{~Hz})$ and beta $(13.0-19.5 \mathrm{~Hz})$ frequency bands and lower values in alpha (8.0$12.5 \mathrm{~Hz}$ ) frequency band than children with adequate academic performance.
Neurofeedback (NFB) has been demonstrated to be an effective treatment in RD children by reinforcing theta/alpha ratio reduction. Its efficacy is based on a cognitive-behavioral improvement and normalization of the EEG frequency.

The aim of this research is to explore if this neurofeedback protocol produces not only a reorganization of the EEG frequency but also a reorganization of EEG connectivity (coherence measures) in children with RD. Ten subjects between the ages of 8 and 10 years were evaluated by structured psychological interview, psychometric, neuropsychological tests, EEG at resting state, and neuropediatric evaluation. All subjects fulfilled the criteria for RD diagnosis and presented a delay of EEG maturation. Participants received NRA treatment using the NFB protocol to decrease theta/alpha ratio. The treatment consisted of thirty 30-min sessions, at a frequency of three times per week. Significant differences were found when preversus posttreatment comparisons were performed. These differences included theta/alpha ratio decrease, an improvement in reading comprehension, and a reduction of the interhemispheric frontal coherence.

Conclusions. These preliminary results demonstrate that NFB treatment, in addition to having an impact on the EEG frequency and the school performance of children with $\mathrm{RD}$, has a positive effect on the EEG coherence values.

Acknowledgements. Héctor Belmont, María Elena Juárez, Nuri Aranda, Saulo Hernández, Teresa Álvarez, Funding: CONACYT-251309 and PAPIITIN207520.

\section{References}

American Psychiatric Association. (2013). Diagnostic and statistical manual of mental disorders (DSM-5). Washington, DC: Author.

Arns, M., Peters, S., Breteler, R., \& Verhoeven, L. (2007). Different brain activation patterns in dyslexic children: Evidence from EEG power and coherence patterns for the double-deficit theory of dyslexia. Journal of Integrative Neuroscience, 6(1), 175-190. https://doi.org/10.1142 IS0219635207001404

Hammond, D. C. (2006). What is neurofeedback? Journal of Neurotherapy, 10(4), 25-36. https://doi.org/10.1300 /J184v10n04 04

Harmony, T., Hinojosa, G., Marosi, E., Becker, J., Rodriguez, M., Reyes, A., \& Rocha, C. (1990). Correlation between EEG spectral parameters and an educational evaluation. International Journal of Neuroscience, 54(1-2), 147-155. https://doi.org/10.3109/00207459008986630

Marosi, E., Harmony, T., Becker, J., Reyes, A., Bernal, J., Fernández, T., ‥ Guerrero, V. (1995). Electroencephalographic coherences discriminate between 
children with different pedagogical evaluation. International Journal of Psychophysiology, 19(1), 23-32. https://doi.org /10.1016/0167-8760(94)00059-N

Vysata, O., Kukal, J., Prochazka, A., Pazdera, L., Simko, J., \& Valis, M. (2014). Age-related changes in EEG coherence. Neurologia i Neurochirurgia Polska, 48(1), 35-38. https://doi.org/10.1016/j.pjnns.2013.09.001

\section{Evaluating the Effect of Cranial Electrotherapy Stimulation on Attention and Sleep in ADHD Erika Rachel Skerrett, Genna Mulvey, Victor Landry, and Genomary Krigbaum \\ Grand Canyon University, Phoenix, Arizona, USA}

This quantitative double-blind, pretest-posttest experimental study examined two research questions in a sample of adults diagnosed with ADHD in the Niagara Region of Ontario, Canada. The questions were: If cranial electrotherapy stimulation (CES) increases auditory and visual attention on the IVA-FSAQ, to what extent does it? And, if CES increases sleep quality on the PSQI, to what extent does it? Sleep problems are common in individuals with ADHD (Sciberras et al., 2017; Wajszilber, Santiseban, \& Gruber, 2018). First line treatment for ADHD has been primarily pharmacological (Cortese et al., 2018). Impaired sleep also causes problems with EF (Wilckens, Woo, Kirk, Erickson, \& Wheeler, 2014). There was a gap in knowledge regarding the linkages between attention and disordered sleep. Understanding the impact of CES on attention and sleep quality was worthy of further investigation. Fifteen participants were randomly assigned to sham or active device conditions and used CES for 60 min each day for 5 weeks. A two-way mixed MANOVA demonstrated a statistically significant multivariate effect for the main effect of time $F(2,12)=7.09, p=.009$; Wilks' $=.541$. There was no statistically significant multivariate effect for the main effect of device group or for the interaction between time and device group. Effect sizes were large to very large, and it is likely that a larger sample size would have revealed more conclusive results regarding the interaction between time and device group. Qualitative data assisted to explain the findings. Sham participants engaged in extraneous activities that could improve sleep during the study. Results support the use of CES as an adjunctive sleep aid in adults suffering from ADHD.

\section{References}

Barclay, T. H., \& Barclay, R. D. (2014). A clinical trial of cranial electrotherapy stimulation for anxiety and comorbid depression. Journal of Affective Disorders, 164, 171-177. https://doi.org/10.1016/j.jad.2014.04.029

Cortese, S., Adamo, N., Del Giovane, C., Mohr-Jensen, C., Hayes, A. J., Carucci, S., ... Cipriani, A. (2018). Comparative efficacy and tolerability of medications for attention-deficit hyperactivity disorder in children, adolescents, and adults: A systematic review and network meta-analysis. Lancet Psychiatry, 5(9), 727-738. https://doi.org/10.1016/S22150366(18)30269-4

Díaz-Román, A., Mitchell, R., \& Cortese, S. (2018). Sleep in adults with ADHD: Systematic review and meta-analysis of subjective and objective studies. Neuroscience \& Biobehavioral Reviews, 89, 61-71. https://doi.org/10.1016 /j.neubiorev.2018.02.014

Faraone, S. V., Po, M. D., Komolova, M., \& Cortese, S. (2019). Sleep-associated adverse events during methylphenidate treatment of attention-deficit/hyperactivity disorder: A metaanalysis. The Journal of Clinical Psychiatry, 80(3). https://doi.org/10.4088/jcp.18r12210

Kennerly, R. C. (2006). Changes in quantitative EEG and lowresolution tomography following cranial electrotherapy stimulation (Doctoral dissertation). Denton, TX: University of North Texas Libraries, UNT Digital Library. Retrieved from http://digital.library.unt.edu/ark:/67531/metadc5364/

Lanius, R. A., Frewen, P. A., Tursich, M., Jetly, R., \& McKinnon, M. C. (2015). Restoring large-scale brain networks in PTSD and related disorders: A proposal for neuroscientificallyinformed treatment interventions. European Journal of Psychotraumatology, 6. https://doi.org/10.3402/ejpt.v6.27313

Lee, J., Lee, H., \& Park, W. (2019). Effects of cranial electrotherapy stimulation on electrocephalogram. Journal of International Academy of Physical Therapy Research, 10(1), 1687-1694.

Schroeder, M. J., \& Barr, R. E. (2001). Quantitative analysis of the electroencephalogram during cranial electrotherapy stimulation. Clinical Neurophysiology, 112(11), 2075-2083. https://doi.org/10.1016/S1388-2457(01)00657-5

Sciberras, E., Mulraney, M., Heussler, H., Rinehart, N., Schuster, T., Gold, L., ... Hiscock, H. (2017). Does a brief, behavioural intervention, delivered by paediatricians or psychologists improve sleep problems for children with ADHD? Protocol for a cluster-randomised, translational trial. BMJ Open, 7(4), e014158. https://doi.org/10.1136/bmjopen-2016-014158

Snitselaar, M. A., Smits, M. G., van der Heijden, K. B., \& Spijker, J. (2017). Sleep and circadian rhythmicity in adult ADHD and the effect of stimulants: A review of the current literature. Journal of Attention Disorders, 21(1), 14-26. https://doi.org $/ 10.1177 / 1087054713479663$

Southworth, S. (1999). A study of the effects of cranial electrical stimulation on attention and concentration. Integrative Physiological and Behavioral Science, 34(1), 43-53. https://doi.org/10.1007/bf02688709

Wajszilber, D., Santiseban, J. A., \& Gruber, R. (2018). Sleep disorders in patients with ADHD: impact and management challenges. Nature and Science of Sleep, 10, 453-480. https://doi.org/10.2147/NSS.S163074

Wilckens, K. A., Woo, S. G., Kirk, A. R., Erickson, K. I., \& Wheeler, M. E. (2014). The role of sleep continuity and total sleep time in executive function across the adult lifespan. Psychology and Aging, 29(3), 658-665. https://doi.org/10.1037/a0037234

Yoon, S. Y. R., Jain, U. R., \& Shapiro, C. M. (2013). Sleep and daytime function in adults with attention-deficit/hyperactivity disorder: Subtype differences. Sleep Medicine, 14(7), 648655. https://doi.org/10.1016/j.sleep.2013.03.003 


\section{Evoked and Induced Gamma Oscillations as Biomarkers of Transcranial Magnetic Stimulation Outcomes in Children with Autism Spectrum Disorder \\ Manuel Casanova', Mohamed Shaban ${ }^{2}$, Mohammed Ghazal ${ }^{3}$, Ayman El-Baz ${ }^{4}$, Emily Casanova ${ }^{1}$, Estate \\ Sokhadze \\ ${ }^{1}$ University of South Carolina School of Medicine-Greenville, Greenville, South Carolina, USA \\ 'University of South Alabama, Mobile, Alabama, USA \\ ${ }^{3}$ University of Abu Dhabi, United Arab Emirates \\ ${ }^{4}$ University of Louisville, Louisville, Kentucky, USA}

Autism spectrum disorder (ASD) is a behaviorally diagnosed neurodevelopmental condition of unknown pathology. Research suggests that abnormalities of brainwave oscillations may provide a biomarker to the condition. More specifically, irregularities of gamma oscillation have been offered as a correlate to the perceptual and cognitive impairments observed in ASD. In this study, evoked and induced gamma frequency oscillations in response to a visual classification task (oddball test with Kanizsa figures) were analyzed and compared in 19 ASD (ADI-R diagnosed, $14.2 \pm 3.61$ years old, 5 girls) and 19 (14.8 \pm 3.67 years old, 5 girls) age/gender matched neurotypical individuals. The ASD group was treated with low frequency rTMS (1.0 Hz, 90\% motor threshold, 18 weekly sessions) targeting the dorsolateral prefrontal cortex (DLPFC). Within any given burst, gamma oscillations do not resemble sinusoidal waveforms of steady amplitude. Instead, their amplitude fluctuates with a variability readily detected by envelope analysis. In this study, we have found that demodulation of gamma oscillations provides information of clinical relevance in the spectral analysis of brainwaves. In autistic subjects, as compared to neurotypicals, significant differences in event-related evoked and induced gamma oscillations were evident in a higher ascending and descending slope magnitude of both evoked and induced gamma oscillation waveforms pre-TMS, especially in response to nontarget cues. In addition, recordings after TMS treatment in our autistic subjects revealed a significant reduction of gamma responses to task-irrelevant stimuli. Participants committed less errors during post-TMS test. Behavioral questionnaires (ABC, RBS-R) showed decrease of irritability, hyperactivity, and repetitive behaviors scores. The use of a novel metric for event-related gamma oscillations (i.e., envelope analysis using wavelet transformation) and measurements of its decay allowed us to characterize the impedance of the originating neuronal circuit. The dampening of gamma oscillations is dependent on the inhibitory tone generated by networks of interneurons. The results suggest that the decay of gamma oscillations may provide a putative biomarker reflective of the excitatory/inhibitory balance of the cortex and a putative outcome measure for TMS-based neuromodulation interventions in autism.

\section{References}

Brown, C. C., Gruber, T., Boucher, J., Rippon, G., \& Brock, J. (2005). Gamma abnormalities during perception of illusory figures in autism. Cortex, 41(3), 364-376. https://doi.org/10.1016/s0010-9452(08)70273-9

Buzsáki, G., \& Wang, X.-J. (2012). Mechanisms of gamma oscillations. Annual Review of Neuroscience, 35, 203-225. https://doi.org/10.1146/annurev-neuro-062111-150444

Casanova, M. F., Sokhadze, E., Opris, I., Wang, Y., \& Li, X (2015). Autism spectrum disorders: Linking neuropathological findings to treatment with transcranial magnetic stimulation. Acta Paediatrica, 104(4), 346-355. https://doi.org/10.1111 /apa.12943

Kanizsa, G. (1976). Subjective contours. Scientific American, 234(4), 48-52. https://doi.org/10.1038 /scientificamerican0476-48

Oberman, L. M., Enticott, P. G., Casanova, M. F., Rotenberg, A., Pascual-Leone, A., McCracken, J. T., \& the TMS in ASD Consensus Group. (2016). Transcranial magnetic stimulation in autism spectrum disorder: Challenges, promise, and roadmap for future research. Autism Research, 9(2), 184203. https://doi.org/10.1002/aur.1567

Rojas, D. C., \& Wilson, L. B. (2014). Gamma-band abnormalities as markers of autism spectrum disorders. Biomarkers in Medicine, 8(3), 353-368. https://doi.org/10.2217/bmm.14.15

Sokhadze, E. M., El-Baz, A. S., Sears, L. L., Opris, I., \& Casanova, M. F. (2014). rTMS neuromodulation improves electrocortical functional measures of information processing and behavioral responses in autism. Frontiers in Systems Neuroscience, $\quad 8, \quad 134$. https://doi.org/10.3389 /fnsys.2014.00134

Uzunova, G., Pallanti, S., \& Hollander, E. (2016). Excitatory/inhibitory imbalance in autism spectrum disorders: implications for interventions and therapeutics. The World Journal of Biological Psychiatry, 17(3), 174-186. https://doi.org/10.3109/15622975.2015.1085597

\section{Feasibility Study of Ambulatory Monitoring of Autonomic Activity in Girls With Rett Syndrome Caroline Buchanan ${ }^{1}$, Jennifer Stallworth ${ }^{1}$, Alan Percy², and Estate Sokhadze \\ ${ }^{1}$ Greenwood Genetic Center, Greenwood, South Carolina, USA \\ ${ }^{2}$ University of Alabama, Birmingham, Alabama, USA \\ ${ }^{3}$ University of South Carolina School of Medicine-Greenville, Greenville, South Carolina, USA}

Rett syndrome (RTT) is a severe but rare neurodevelopmental disorder caused by pathogenic variants in the methyl CpG-binding protein 2 gene (MECP2) occurring in 1 of 10,000 girls (Neul et al., 2010). Evaluation of autonomic activity in children with RTT is challenging due to stereotyped behaviors presenting with repetitive involuntary hand movements. According to parental reports, sleep disturbances and a range of emotional, behavioral, 
and autonomic dysregulation present in children with RTT are the main factors affecting their quality of life (Boban, Leonard, Wong, Wilson, \& Downs, 2018; Killian et al., 2016; Lane et al., 2011). Autonomic dysfunction is likely to have a significant functional impact on such symptoms as repetitive rocking, screaming, agitation and anxiety (Carroll, Ramirez, \& Weese-Mayer, 2020; Kumar et al., 2017; Singh, Lanzarini, \& Santosh, 2019; Weese-Mayer et al., 2008). The study examined the feasibility of using an E4 ambulatory autonomic monitoring device (Empatica, Boston, MA) to evaluate heart rate (HR), electrodermal activity (EDA) and skin temperature (TEMP) during 24 to 72 hours recording in girls with confirmed RTT ( $N=10$, 3-12 years old, mean 6.3 years). Caregivers were instructed to place the E4 device on their child's wrist and record sessions for at least 24 hours, and, if possible, to have several sessions recorded. An array of HR, EDA, and TEMP measures were evaluated from the collected 26 qualifying overnight and daytime in-home ambulatory recordings in 10 girls with RTT. Although hand stereotypies are present in the majority of children with RTT, they do not occur during sleep (Merbler, Byiers, Garcia, Feyma, \& Symons, 2018), and the intensity of hand movements varies. Examinations of recordings allowed separation of data into three conditions according to movement intensity assessed by a built-in accelerometer of the E4 device: sleep and day nap (Zero), low level (Low) and high level (High) of hand stereotypies. After debugging of movementrelated artifacts in HR, EDA, and TEMP, the segments selected according to movement activity states (Zero, Low, High) were averaged according to condition and compared using paired sample $t$-test. Statistical analysis of EDA showed significant differences between Zero and Low conditions $(t=$ 2.42, $p=.036)$ and trend to lower EDA in High as compared to Low activity $(t=2.20, p=.052)$. TEMP was significantly higher during sleep as compared to both waking conditions $(p<.001)$, while HR was lower in Zero as compared to both Low $(t=5.64, p<$ $.001)$ and High $(t=5.11, p=.001)$ intensity movement conditions. Recording of EDA in individuals with RTT is very rare and was reported only for pain experienced during blood draw (O'Leary et al., 2017). Recording EDA from the E4 sensors placed on the wrist had lower absolute values but was informative to detect dynamics during sleep and stereotyped movements. In future studies, we plan to correlate autonomic measures with behavioral questionnaire scores and clinical severity in each subject with RTT. Our results confirm feasibility of in-home physiological recording and ability to detect variations of autonomic measures in girls with RTT. Study was supported by the International Rett Syndrome Foundation grant.

\section{References}

Boban, S., Leonard, H., Wong, K., Wilson, A., \& Downs, J. (2018). Sleep disturbances in Rett syndrome: Impact and management including use of sleep hygiene practices. American Journal of Medical Genetics. Part A, 176(7), 15691577. https://doi.org/10.1002/ajmg.a.38829

Carroll, M. S., Ramirez, J.-M., \& Weese-Mayer, D. E. (2020). Diurnal variation in autonomic regulation among patients with genotyped Rett syndrome. Journal of Medical Genetics, 57(11), 786-793. https://doi.org/10.1136/jmedgenet-2019106601

Killian, J. T., Lane, J. B., Lee, H.-S., Pelham, J. H., Skinner, S. A., Kaufmann, W. E., ... Percy, A. K. (2016). Caretaker quality of life in Rett Syndrome: Disorder features and psychological predictors. Pediatric Neurology, 58, 67-74. https://doi.org /10.1016/j.pediatrneurol.2015.12.021

Kumar, A., Jaryal, A., Gulati, S., Chakrabarty, B., Singh, A., Deepak, K. K., ... Khajuria, R. (2017). Cardiovascular autonomic dysfunction in children and adolescents with Rett syndrome. Pediatric Neurology, 70, 61-66. https://doi.org /10.1016/j.pediatrneurol.2017.01.010

Lane, J. B., Lee, H.-S., Smith, L. W., Cheng, P., Percy, A. K., Glaze, D. G., ... Krischer, J. P. (2011). Clinical severity and quality of life in children and adolescents with Rett syndrome. Neurology, $77(20), \quad 1812-1818 . \quad$ https://doi.org/10.1212 /WNL.0b013e3182377dd2

Merbler, A. M., Byiers, B. J., Garcia, J. J., Feyma, T. J., \& Symons, F. J. (2018). The feasibility of using actigraphy to characterize sleep in Rett syndrome. Journal of Neurodevelopmental Disorders, 10(1), 8. https://doi.org /10.1186/s11689-018-9227-z

Neul, J. L., Kaufmann, W. E., Glaze, D. G., Christodoulou, J., Clarke, A. J., Bahi-Buisson, N., ... Rett Search Consortium. (2010). Rett syndrome: Revised diagnostic criteria and nomenclature. Annals of Neurology, 68(6), 944-950. https://doi.org/10.1002/ana.22124

O'Leary, H. M., Marschik, P. B., Khwaja, O. S., Ho, E., Barnes, K. V., Clarkson, T. W., ... Kaufmann, W. E. (2017). Detecting autonomic response to pain in Rett syndrome. Developmental Neurorehabilitation, 20(2), 108-114. https://doi.org/10.3109 /17518423.2015.1087437

Singh, J., Lanzarini, E., \& Santosh, P. (2019). Autonomic dysfunction and sudden death in patients with Rett syndrome: A systematic review. Journal of Psychiatry \& Neuroscience, 45(1), 150-181. https://doi.org/10.1503/jpn.190033

Weese-Mayer, D. E., Lieske, S. P., Boothby, C. M., Kenny, A. S., Bennett, H. L., \& Ramirez, J.-M. (2008). Autonomic dysregulation in young girls with Rett Syndrome during nighttime in-home recordings. Pediatric Pulmonology, 43(11), 1045-1060. https://doi.org/10.1002/ppul.20866

\section{Individualized Neurofeedback Treatment for Anxiety and Related Symptoms \\ J. Ross Spears, Cerise Edmonds, J. Claire Gregory, Devon Romero, and Mark Jones \\ University of Texas at San Antonio, San Antonio, Texas, USA}

Working within the mental health profession, clinicians might encounter individuals who struggle with various anxiety symptoms. Anxiety has a variety of forms. Anxiety can negatively impede on 
daily functions and roles. Existing in research are several approaches using biofeedback as an intervention for treating anxiety (Jones \& Hitsman, 2018). Neurofeedback, a facet of biofeedback, reveals itself as a promising avenue for treating and correcting unwanted symptoms of anxiety as well as other diagnoses (Cheon et al., 2015; Kerson, Sherman, \& Kozlowski, 2009; Mennella, Patron, \& Palomba, 2017; Scheinost et al., 2013; Wang et al., 2019; Zhao et al., 2018).

Our poster presentation will present findings from our retrospective study that integrated a withinsubjects research design using individualized neurofeedback protocols for anxiety. This presentation has three primary aims. The first is to disseminate findings from our retrospective study that examined the effects of quantitative electroencephalography (qEEG) guided amplitude neurofeedback treatment for anxiety and related symptoms. The current sample includes fifty-two adult clients assessed for anxiety using qEEG and symptom measures (Zung Self-Rating Anxiety Scale [SAS] and the Achenbach System of Empirically Based Assessment Adult Self-Report [ASR]) collected pre- and post-intervention. Clients $(N=$ 52) range in age from 19 to $59(M=36.4, S D=12.6)$ with $53.8 \%(n=28)$ of clients self-reporting as male. The self-reported ethnic composition of the sample was $50 \%(n=26)$ Non-Hispanic, $44 \%(n=23)$ Hispanic/Latino, and $6 \%(n=3)$ chose not to respond. On the SAS, for all subjects, the mean of the pre-scores was $45.62(S D=8.49)$ and the mean of the post-scores was $39.50(S D=9.40)$. A paired samples $t$-test yielded a statistically significant improvement, with $t(51)=6.3, p<.001, d=1.11$. On the ASR, a statistically significant improvement was also measured in seven of the eight syndrome scales. In addition, a statistically significant improvement was measured for total problems and critical items. The second aim is to discuss predictors of SAS outcomes assessed in the first aim. Regression analyses identified total problems, substance use, and level of education as predictors of anxiety symptoms as measured by the SAS following individualized neurofeedback treatment. Finally, our third aim is to present findings from a subsample of these clients with assessments completed at three time points (pre, post, and followup). This subsample consisted of 21 clients with ages ranging from 20 to $56(M=38.8, S D=12.39)$. Analyses revealed statistically significant improvement from pre to post and sustained outcomes from post to follow-up. Implications of these findings and future research will be discussed.

\section{References}

Cheon, E.-J., Koo, B.-H., Seo, W.-S., Lee, J.-Y., Choi, J.-H., \& Song, S.-H. (2015). Effects of neurofeedback on adult patients with psychiatric disorders in a naturalistic setting. Applied Psychophysiology and Biofeedback, 40(1), 17-24. https://doi.org/10.1007/s10484-015-9269-x

Jones, M. S., \& Hitsman, H. (2018). QEEG-guided neurofeedback treatment for anxiety symptoms. NeuroRegulation, 5(3), 8592. http://dx.doi.org/10.15540/nr.5.3.85

Kerson, C., Sherman, R. A., \& Kozlowski, G. P. (2009). Alpha suppression and symmetry training for generalized anxiety symptoms. Journal of Neurotherapy, 13(3), 146-155. https://doi.org/10.1080/10874200903107405

Mennella, R., Patron, E., \& Palomba, D. (2017). Frontal alpha asymmetry neurofeedback for the reduction of negative affect and anxiety. Behaviour Research and Therapy, 92, 32-40. https://doi.org/10.1016/j.brat.2017.02.002

Scheinost, D., Stoica, T., Saksa, J., Papademetris, X., Constable, R. T., Pittenger, C., \& Hampson, M. (2013). Orbitofrontal cortex neurofeedback produces lasting changes in contamination anxiety and resting-state connectivity. Translational Psychiatry, 3(4), e250. https://doi.org /10.1038/tp.2013.24

Wang, S.-Y., Lin, I.-M., Fan, S.-Y., Tsai, Y.-C., Yen, C.-F., Yeh, Y.-C., ... Lin, H.-C. (2019). The effects of alpha asymmetry and high-beta down-training neurofeedback for patients with the major depressive disorder and anxiety symptoms. Journal of Affective Disorders, 257, 287-296. https://doi.org /10.1016/j.jad.2019.07.026

Zhao, Z., Yao, S., Li, K., Sindermann, C., Zhou, F., Zhao, W., ... Becker, B. (2018). Real-time functional connectivity-based neurofeedback of amygdala-frontal pathways reduces anxiety. bioRxiv, 308924. https://doi.org/10.1101/308924

\section{Neurofeedback Training for Improving Attention in Amphetamine Addict \\ Hsin-Yi Chiang ${ }^{1}$, Hsueh-Chen Lu \\ ${ }^{1}$ Kaohsiung Municipal Kai-Syuan Psychiatric Hospital, Kaoshiung City, Taiwan \\ ${ }^{2}$ California School of Professional Psychology, Alliant International University, California, USA}

Introduction. Amphetamine dependence was found to be associated with poorer performance on attention/concentration and ability to discriminate targets from nontargets on the Continuous Performance Test (CPT; Ersche \& Sahakian, 2007; McKetin \& Mattick, 1997). Eyes-open betasensorimotor response (SMR) amplitude uptraining is known to be an effective way to improve focusing on attentional ability (Hong \& Lee, 2012). This case study investigated the effects of beta-SMR neurofeedback training on attention in a patient who is a chronic amphetamine user.

Case Description. The patient is a 53-year-old Taiwanese female who had been addicted to amphetamine for at least 30 years and suffered chronic spondylolisthesis pain for over 7 years. She reported using amphetamine to manage her pain and improve her work performance. However, her 
amphetamine dependence problem and chronic pain contributed to her depression, anxiety, insomnia, impairments at work, and reduction of her ability to work. She had not received medication management for her problems with amphetamine dependence, mental health problems, and chronic pain. She was referred from an outpatient psychiatric program to receive beta-SMR neurofeedback training combined with relaxation training to improve her mental health problems, substance use, and chronic pain issues. She received beta-SMR neurofeedback training at the central area $(\mathrm{Cz})$ to increase her beta and SMR amplitude twice per week for 2 months (1 hour/time), and she practiced diaphragmatic breathing relaxation skills to improve her emotional and sleep problems. Her attention, impulse control, and emotional problems were assessed pre- and postneurofeedback training with Conners' Continuous Performance Test-II (CPT-II), Adult ADHD Self-Report Scale (ASRS), the Beck Depression Inventory-II (BDI-II), and the Beck Anxiety Inventory (BAI). After 2 months (12 hours) of beta-SMR neurofeedback training, her attention problems, impulsivity, depression, anxiety, and subjective experience of chronic pain all showed clinically significant improvement.

Discussion. The present case study supports the efficacy of a beta-SMR neurofeedback training as an adjunctive therapy in an outpatient drug treatment program, and supportive data were provided through attention and psychological assessments. Future research should include multiple cases to examine the efficacy of beta-SMR neurofeedback training for chronic amphetamine users.

\section{References}

Ersche, K. D., \& Sahakian, B. J. (2007). The neuropsychology of amphetamine and opiate dependence: Implications for treatment. Neuropsychology Review, 17(3), 317-336. https://dx.doi.org/10.1007/s11065-007-9033-y

Hong, C., \& Lee, I. (2012). Effects of neurofeedback training on attention in children with intellectual disability. Journal of Neurotherapy, 16(2), 110-122. https://doi.org/10.1080 /10874208.2012.677666

McKetin, R., \& Mattick, R. P. (1997). Attention and memory in illicit amphetamine users. Drug and Alcohol Dependence, 48(3), 235-242. https://doi.org/10.1016/s03768716(97)00132-4
Neuromodulation of Event-Related Gamma Oscillations in Children with Autism

Estate Sokhadze ${ }^{1}$, Mohamed Shaban ${ }^{2}$, Mohhamed Ghazal $^{3}$, Ayman El-Baz ${ }^{4}$, and Manuel Casanova ${ }^{5}$

'University of South Carolina School of Medicine-Greenville, Greenville, South Carolina, USA

${ }^{2}$ University of South Alabama, Mobile, Alabama, USA

${ }^{3}$ University of Abu Dhabi, United Arab Emirates

${ }^{4}$ University of Louisville, Louisville, Kentucky, USA

${ }^{5}$ University of South Carolina School of Medicine-Greenville, Greenville, South Carolina, USA

Oscillatory activity in the gamma band of the EEG has been related to cognitive functions such as attention, learning, and memory (Kaiser \& Lutzenberger, 2003; Kahana, 2006). Abnormalities of high frequency EEG oscillations in gamma range have been associated with binding problems present in autism and other psychiatric conditions (Brock, Brown, Boucher, \& Rippon, 2002). Several lines of evidence suggest that the cortical excitatory/inhibitory (E/I) imbalance observed in autism spectrum disorder (ASD) is the result of an inhibitory deficit. An abnormal E/l bias provides a pathophysiological mechanism capable of explaining the complex phenotype of ASD. Abnormalities of E/I ratio in ASD were discussed in several reviews (Rubenstein \& Merzenich, 2003; Uzunova, Pallanti, \& Hollander, 2016). Gamma-band abnormalities have been reported in many studies of autism (Rojas \& Wilson, 2014) and were often associated with perceptual and cognitive functions that are compromised in autism. Low frequency repetitive transcranial magnetic stimulation (rTMS) over the dorsolateral prefrontal cortex (DLPFC) has been proven to normalize gamma oscillation abnormalities, executive functions, and repetitive behaviors in high functioning (ASD) individuals (Oberman et al., 2016; Sokhadze et al., 2016). In this study, gamma frequency oscillations in 35-45 $\mathrm{Hz}$ range in response to task-relevant and taskirrelevant illusory Kanizsa figures (Kanizsa, 1976) were analyzed and compared in 19 ASD (ADI-R diagnosed, $14.2 \pm 3.61$ years old) and 19 (14.8 \pm 3.67 years old) gender-matched neurotypical children. The ASD group was treated with 18 weekly $1.0 \mathrm{~Hz}$ frequency rTMS $(90 \%$ motor threshold). In autistic children, as compared to neurotypicals, at the baseline test, significant differences in event-related gamma oscillations were evident at the frontal sites (F3, F4, F7, F8) in a form of a higher amplitude to both rare target (e.g., at F3 EEG site, by $0.92 \pm 1.17 \mathrm{uV}, p<.01)$ and rare nontarget illusory figures $(1.05 \pm 1.01 \mathrm{uV}, p<.001)$. Recordings of event-related gamma oscillations after TMS treatment in our autistic subjects revealed a significant reduction in the latency of positive 
ascending slope of gamma oscillation peak along with an increase in latency of the negative descending slope of the gamma oscillation burst. Differences between latencies of ascending and descending slopes post-TMS course decreased in response to all type stimuli. The results were interpreted as reflective of increased inhibitory tone suggesting that low frequency rTMS treatment course in autism normalizes the gamma oscillations by increasing the inhibitory tone of the cortex. The present study contributes to a proposal that beneficial effects of rTMS in behavioral outcomes could be mediated by improvements in gamma band $(35-45 \mathrm{~Hz})$ oscillatory activity. The results suggest that the analysis of various metrics of gamma oscillations may serve as a basis for development of biomarkers of the excitatory/inhibitory balance of the cortex and provide with valuable outcome measures for neuromodulation-based interventions in autism.

\section{References}

Brock, J., Brown, C. C., Boucher, J., \& Rippon, G. (2002). The temporal binding deficit hypothesis of autism. Developmental and Psychopathology, 14(2), 209-224. https://doi.org/10.1017 /s0954579402002018

Kaiser, J., \& Lutzenberger, W. (2003). Induced gamma-band activity and human brain function. The Neuroscientist, 9(6), 475-484. https://doi.org/10.1177/1073858403259137

Kahana, M. J. (2006). The cognitive correlates of human brain oscillations. The Journal of Neuroscience, 26(6), 1669-1672. https://doi.org/10.1523/JNEUROSCI.3737-05c.2006

Kanizsa, G. (1976). Subjective contours. Scientific American, 235, $48-52$.

Oberman, L. M., Enticott, P. G., Casanova, M. F., Rotenberg, A., Pascual-Leone, A., McCracken, J. T., \& the TMS in ASD Consensus Group. (2016). Transcranial magnetic stimulation in autism spectrum disorder: Challenges, promise, and roadmap for future research. Autism Research, 9(2), 184203. https://doi.org/10.1002/aur.1567

Rubenstein, J. L. R., \& Merzenich, M. M. (2003). Model of autism: Increased ratio of excitation/inhibition in key neural systems. Genes, Brain, and Behavior, 2(5), 255-267. https://doi.org/10.1034/j.1601-183x.2003.00037.x

Rojas, D. C., \& Wilson, L. B. (2014). Gamma-band abnormalities as markers of autism spectrum disorders. Biomarkers Medicine, 8(3), 353-368. https://doi.org/10.2217/bmm.14.15

Sokhadze, E. M., Casanova, M. F., El-Baz, A. S., Farag, H. E., Li, X., \& Wang, Y. (2016). TMS-based neuromodulation of evoked and induced gamma oscillations and event-related potentials in children with autism. NeuroRegulation, 3(3), 101-126. https://doi.org/10.15540/nr.3.3.101

Uzunova, G., Pallanti, S., \& Hollander, E. (2016). Excitatory/inhibitory imbalance in autism spectrum disorders: Implications for interventions and therapeutics. The World Journal of Biological Psychiatry, 17(3), 174-186. https://doi.org/10.3109/15622975.2015.1085597
Posttraining Registration of an SMR/theta Protocol with Neurofeedback

Legna Alejandra Lazo Sánchez ${ }^{1}$, Fructuoso Ayala Guerrero', and Diana Amparo Martínez Huerta²

${ }^{1}$ Faculty of Psychology, National Autonomous University of Mexico, Mexico City, Mexico

${ }^{2}$ Boston Neurodynamics, Brookline, Massachusetts, USA

The literature reports that it is possible to modify the pattern of electroencephalographic (EEG) activity using neurofeedback techniques; however, such findings continue to have limitations. One of the most widely used clinical protocols is to increase the sensorimotor rhythm (SMR) and simultaneously decrease theta activity with the aim of increasing attention performance and reducing hyperactive and impulsive behaviors. The SMR band is characterized by a frequency of $12-15 \mathrm{~Hz}$ and is the expression of synchronized oscillatory activity, reflected in the sensory motor cortex; it is associated with body movement and concentration capacity (Gruzelier, Inoue, Smart, Steed, \& Steffert, 2010). The neurogenesis of SMR emanates from the ventrobasal nucleus of the thalamus, which is generally associated with conduction of broad somatosensory information. During the production of conditioned SMR, the firing patterns of the ventrobasal nucleus change from rapid, nonrhythmic (tonic) discharges to bursts of rhythmic and systematic discharges, which in turn are associated with suppression of somatosensory information passing (Sterman \& Egner, 2006). Theta waves have a frequency of $4-8 \mathrm{~Hz}$ and are considered slow activity waves that are involved with sleep as well as with some dysfunctional mechanisms such as distraction and inattention (Koudelková \& Strmiska, 2018; Demos, 2005). This is a pilot study of a doctoral thesis. The purpose of this study was to evaluate the efficacy of SMR/theta training in neurofeedback through a posttraining registry where the participant evokes the trained rhythms without looking at the screen after 25 training sessions in five healthy volunteers. In this study we worked with five healthy volunteer participants between 21 and 30 years old. The neurofeedback protocol SMR/theta used consisted of 25 sessions lasting 30 min. Subsequently, a posttraining registry was carried out to evaluate the learning obtained after the protocol. Participants were asked to replicate the learned brain state without having access to the feedback screen. With the following conditions: Closed eyes, Opened eyes, Training 1, Rest 1, Training 2, and Rest 2. During the posttraining registration, significant differences were obtained in the average amplitudes between each of the recording phases for the theta frequency band, decreasing when the target task was performed and 
increasing during the rest phase specifically between Training 1 and Rest $1, Z(1,5)=-2,023, p=$ .043; as well as Training 2 and Rest $2, Z(1,5)=$ $-2,023, p=.043$. In the case of electrical activity SMR no significant differences were found between the different phases of the records; however, it is important to note that the trend of the data kept increasing throughout the entire record. The behavior of the SMR and theta wave patterns is as expected according to the reported literature (Aliño Costa, 2017; de Zambotti, Bianchin, Magazzini, Gnesato, \& Angrilli, 2012).

\section{References}

Aliño Costa, M. (2017). Entrenamiento asistido por neurofeedback: Análisis de los efectos psicofisiológicos inmediatos y factores de personalidad influyentes (Doctoral dissertation, Universitat de València). http://roderic.uv.es /handle/10550/61004

Demos, J., (2005). Getting started with neurofeedback. (1st ed.). New York, NY: W. W. Norton \& Company, Inc.

de Zambotti, M., Bianchin, M., Magazzini, L., Gnesato, G., \& Angrilli, A. (2012). The efficacy of EEG neurofeedback aimed at enhancing sensory-motor rhythm theta ratio in healthy subjects. Experimental Brain Research, 221(1), 69-74. https://doi.org/10.1007/s00221-012-3148-y

Gruzelier, J., Inoue, A., Smart, R., Steed, A., \& Steffert, T. (2010). Acting performance and flow state enhanced with sensorymotor rhythm neurofeedback comparing ecologically valid immersive VR and training screen scenarios. Neuroscience Letters, $\quad 480(2), \quad 112-116 . \quad$ https://doi.org/10.1016 j.neulet.2010.06.019

Koudelková, Z., \& Strmiska, M. (2018). Introduction to the identification of brain waves based on their frequency. MATEC Web of Conferences, 210, 05012. https://doi.org/10.1051/matecconf/201821005012

Sterman, M. B., \& Egner, T. (2006). Foundation and practice of neurofeedback for treatment of epilepsy. Applied Psychophysiology and Biofeedback, 31(1), 21-35. https://doi.org/10.1007/s10484-006-9002-x

\section{Prefrontal Neurofeedback Training Outcomes in Children with Autism Spectrum Disorder with Comorbid Attention-Deficit/Hyperactivity Disorder \\ Estate Sokhadze ${ }^{1}$, Desmond Kelly², and Manuel F. Casanova \\ ${ }^{1}$ University of South Carolina School of Medicine-Greenville, Greenville, South Carolina, USA \\ ${ }^{2}$ Prisma Health-Upstate, Greenville, South Carolina, USA}

Neurofeedback (NFB) is recognized as one of the most established neurotherapy methods in ADHD (Arns et al., 2020) and is considered as the potentially effective method for EEG self-regulation in children with autism spectrum disorder (ASD). There are numerous reports of high comorbidity of these two disorders (Sinzig, Vinzelberg, Evers, \& Lehmkuhl, 2014) and the number of children with $A S D$ and $A D H D$ is increasing after recent changes in DSM-5 that allowed this form of dual diagnosis. It should be noted that most studies of neurofeedback focus on posttreatment behavioral evaluations and EEG outcomes and do not analyze dynamics of EEG and autonomic activity during individual training sessions. Meanwhile, there are certain feasibility issues in continuing NFB in children with dual diagnosis that are considered as nonresponders. This brings on a need for identification of reliable predictors of successful outcomes considering the required number of sessions in the NFB course. Some EEG, cardiorespiratory, and electrodermal indices are candidate predictors of neurofeedback training outcomes and may serve as potential moderators. We proposed that 24 sessions of prefrontal neurofeedback training in treatment responders will be accompanied by intended changes in targeted EEG bands and ratios of individuals bands (e.g., theta/beta ratio), as well as by changes in electrodermal and cardiorespiratory indices. Outcome measures were based on EEG, ECG, pneumogram and skin conductance indices and parental behavioral ratings. The protocol used a training for wide band EEG amplitude suppression (sometimes referred to as "squash") with concurrent upregulation of the $40 \mathrm{~Hz}$-centered gamma. In this pilot study we enrolled 12 children diagnosed both with ASD and ADHD (11.3 years, $S D=1.4,3$ girls). For each session of neurofeedback, qEEG analysis at the training site was completed to determine the relative power of the individual bands (theta, beta, and gamma) and their ratios (theta/beta) within and between sessions. We analyzed Aberrant Behavior Checklist (ABC; Aman \& Singh, 1994) and ASEBA (Achenbach \& Rescorla, 2012) ratings by parents. Nine subjects were defined as responders. Regression analysis revealed significant linear increase of skin conductance level (SCL) along with decrease of respiration rate during each successful neurofeedback session during successful training sessions defined by targeted changes of EEG measures of interest. Heart rate variability (HRV) measures showed certain trends during sessions but did not reach significance level. According to parental reports hyperactivity subscale scores of $A B C$ and ASEBA DSM-oriented scores of attentionrelated problems showed significant decrease. Psychophysiological measures such as SCL, respiration, and HRV represent useful markers of attention and emotional engagement of children with ASD and ADHD during neurofeedback and can be used as predictors of successful performance during sessions and behavioral outcome of the intervention. The study extends our prior studies (Sokhadze, Casanova, Wang, Tasman, \& Kelly, 2019; Wang et al., 2016) using similar protocol by extending 
number of training sessions from 18 to 24 and by adding concurrent psychophysiological monitoring through the course of training and supports rationale for more extensive use of autonomic measures for interpretation of moderators and mediators of neurofeedback intervention.

\section{Supported by GHS Transformative Seed Grant.}

\section{References}

Achenbach, T. M., \& Rescorla, L. A. (2012). Multicultural guide for the ASEBA gorms and profiles for ages $11 / 2-18$ (2nd ed.). Burlington, VT: University of Vermont Research Center for Children, Youth, \& Families.

Aman, M. G., \& Singh, N. N. (1994). Aberrant behavior checklist. Supplementary manual. East Aurora, NY: Slosson Educational Publications.

Arns, M., Clark, C. R., Trullinger, M., deBeus, R., Mack, M., \& Aniftos, M. (2020). Neurofeedback and attentiondeficit/hyperactivity-disorder (ADHD) in children: Rating the evidence and proposed guidelines. Applied Psychophysiology and Biofeedback, 45, 39-48. https://doi.org/10.1007/s10484020-09455-2

Sinzig, J., Vinzelberg, I., Evers, D., \& Lehmkuhl, G. (2014). Executive function and attention profiles in preschool and elementary school children with autism spectrum disorders or ADHD. International Journal of Developmental Disabilities, 60(3), 144-154. https://doi.org/10.1179 2047387714Y.0000000040

Sokhadze, E., Casanova, M. F., Wang, Y., Tasman, A., \& Kelly, D. P. (2019). Effects of the length of prefrontal neurofeedback training in children with autism spectrum disorder. In M. C. Hellinger (Ed.), Neurofeedback: Functions, applications and effects (pp. 77-116). Hauppauge, NY: Nova Science Publishers Inc.

Wang, Y., Sokhadze, E. M., El-Baz, A. S., Li, X., Sears, L., Casanova, M. F., \& Tasman, A. (2016). Relative power of specific EEG bands and their ratios during neurofeedback training in children with autism spectrum disorder. Frontiers in Human Neuroscience, 9, 723. https://doi.org/10.3389 /fnhum.2015.00723

Received: December 3, 2020

Accepted: December 3, 2020

Published: December 29, 2020 